\title{
FOURIER SERIES WITH POSITIVE COEFFICIENTS
}

BY R. P. BOAS, JR. ${ }^{1}$

\author{
Communicated by A. Zygmund, April 22, 1966
}

I shall state a number of results on sine or cosine series with nonnegative coefficients; proofs of these and some related theorems will appear elsewhere.

The following theorems are known.

A [1], [8]. If $\lambda_{n} \downarrow 0, \phi(x)=\sum \lambda_{n} \cos n x$, and $0<\gamma<1$, then $\sum n^{\gamma-1} \lambda_{n}$ $<\infty$ if and only if $x^{-\gamma} \phi(x) \in L$.

$\mathrm{A}^{\prime}$ [6]. If $\lambda_{n}$ are the Fourier coefficients of $\phi, \lambda_{n} \geqq 0$, and $1<\gamma<3$, then $\sum n^{\gamma-1} \lambda_{1}<\infty$ if and only if $x^{-\gamma}[\phi(x)-\phi(0)] \in L$.

B [2], [3]. If $\lambda_{n} \downarrow 0, \phi(x)=\sum \lambda_{n} \cos n x, 1<p<\infty$, and $(1-p) / p$ $<\gamma<1 / p$, then $x^{-\gamma} \phi(x) \in L^{p}$ if and only if $\sum n^{p+p \gamma-2} \lambda_{n}^{p}<\infty$.

C [7]. If $\lambda_{n} \downarrow 0, \phi(x)=\sum \lambda_{n} \cos n x$, and $0<\gamma<1$, then $\phi(x) \in \operatorname{Lip} \gamma$ if and only if $\lambda_{n}=O\left(n^{-\gamma-1}\right)$.

There are similar theorems for sine series.

The following theorems generalize $\mathrm{A}$ and $\mathrm{C}$ (with different necessary and sufficient conditions), to series with nonnegative coefficients, and give a result that is related to $\mathrm{B}$ as $\mathrm{A}^{\prime}$ is related to $\mathrm{A}$.

Theorem 1. If $\lambda_{n} \geqq 0, \lambda_{n}$ are the Fourier sine or cosine coefficients of $\phi$ and $0<\gamma<1$, then

$$
\sum n^{\gamma-1} \lambda_{n}<\infty
$$

if and only if

$$
\int_{a+}^{\pi}(x-a)^{-\gamma} \phi(x) d x \text { converges, } \quad 0 \leqq a<\pi .
$$

More precisely, (1) is necessary for (2) with $a=0$ and sufficient for (2) for all $a$-an illustration of the principle that a Fourier series with nonnegative coefficients tends to behave as well at all points as it does at 0 . (The case $a=0$ is a special case of a more general result of Edmonds [4, p. 235].) Theorem $\mathrm{A}^{\prime}$ can be generalized in the same way if $1<\gamma<2$.

${ }^{1}$ Supported by National Science Foundation Grant GP-3940. 
THEOREM 2. If $\lambda_{n} \geqq 0$ and $\lambda_{n}$ are the Fourier sine or cosine coefficients of $\phi$, and $1 / p<\gamma<(p+1) / p$, then

$$
|x-a|^{-\gamma}|\phi(x)-\phi(a)| \in L^{p}, \quad 0 \leqq a<\pi,
$$

if and only if

$$
\sum_{n=1}^{\infty} n^{p \gamma-2}\left(\sum_{k=n}^{\infty} \lambda_{k}\right)^{p}<\infty .
$$

More precisely, (4) is necessary for (3) if $a=0$ and sufficient for (3) for all $a$. Theorem $\mathrm{B}$ can be obtained as a corollary.

Theorem 3. If $\lambda_{n} \geqq 0, \lambda_{n}$ are the Fourier sine or cosine coefficients of $\phi$, and $0<\gamma<1$, then $\phi \in \operatorname{Lip} \gamma$ if and only if

$$
\sum_{k=n}^{\infty} \lambda_{k}=O\left(n^{-\gamma}\right)
$$

When $\lambda_{k} \downarrow 0,(5)$ is equivalent to $\lambda_{n}=O\left(n^{-1-\gamma}\right)$, so Theorem $\mathrm{C}$ is a corollary. Theorem 3 is formally the limiting case $p=\infty$ of Theorem 2 .

Theorem 3 fails when $\gamma=1$. There are a number of substitutes, among them the following result, in which $\Lambda_{*}$ and $\lambda_{*}$ denote the classes of continuous functions $\phi$ such that $\phi(x+h)+\phi(x-h)-2 \phi(x)$ $=O(h)$ or $o(h)$, uniformly in $x[10, \mathrm{p} .43]$.

Theorem 4. If $\lambda_{n} \geqq 0$ and $\lambda_{n}$ are the Fourier cosine coefficients of $\phi$, then (5) with $\gamma=1$ is a necessary and sufficient condition for either $f(x)-f(0)=O(x)$ or $f \in \Lambda_{*}$;

$$
\sum_{k=n}^{\infty} \lambda_{k}=O\left(n^{-1}\right)
$$

is necessary and sufficient for either $f(x)-f(0)=o(x)$ or $f \in \lambda_{*}$; if (6) holds, then $f^{\prime}(x)$ exists $\left[f^{\prime}\right.$ is continuous] if and only if $\sum k \lambda_{k} \sin k x$ converges [converges uniformly].

Paley (see [5, p. 72]; [9]) showed that if the sine series of a continuous function has nonnegative coefficients then the series converges uniformly. As a corollary of Theorem 4 we have a localization of this.

THEOREM 5. If $\phi$ has nonnegative sine coefficients $\lambda_{n}$ and

$$
\sum_{n}^{\infty} k^{-1} \lambda_{k}=O(1 / n)
$$


then $\sum \lambda_{k} \sin k x$ converges (for any particular $x$ ) if and only if $\phi$ is the derivative of its integral at $x$.

In fact, if $\phi$ is continuous, $\int \phi \in \lambda_{*}$ and so (7) holds.

\section{REFERENCES}

1. R. P. Boas, Jr., Integrability of trigonometric series. III, Quart. J. Math. Oxford Ser. (2) 3 (1952), 217-221.

2. - On the integrability of functions defined by trigonometrical series, Math $Z$. 66 (1956), 9-12.

3. Y.-M. Chen, Some asymptotic properties of Fourier constants and integrability theorems, Math. Z. 68 (1957), 227-244.

4. S. M. Edmonds, The Parseval formulae for monotonic functions. II, Proc. Cambridge Philos. Soc. 46 (1950), 231-248.

5. G. H. Hardy and W. W. Rogosinski, Fourier series, Cambridge Univ. Press, New York, 1944.

6. P. Heywood, On the integrability of functions defined by trigonometric series, Quart. J. Math. Oxford Ser. (2) 5 (1954), 71-76.

7. G. G. Lorentz, Fourier-Koeffizienten und Funktionenklassen, Math. Z. 51 (1948), 135-149.

8. G. Sunouchi, Integrability of trigonometric series, J. Math. Tokyo 1 (1953), 99103.

9. O. Szász, On the partial sums of certain Fourier series, Amer. J. Math. 59 (1937), 696-708.

10. A. Zygmund, Trigonometric series, 2d ed., Vol. 1, Cambridge Univ. Press, New York, 1959.

NORTHWESTERN UNIVERSITY 